\title{
Mit dem Smartphone für die Wissenschaft - Wie Bürger zur Kenntnis der Frankfurter und hessischen Flora beitragen können
}

\author{
Indra Starke-Ottich, Marco Schmidt und Georg Zizka
}

\begin{abstract}
The free app „iNaturalist“ brings interested laymen and experts together. The app provides low-threshold access to biodiversity recording and plant identification, while experts ensure quality assurance. Projects have been initiated on various spatial levels, e.g. by BVNH e.V., the Senckenberg Research Institute and Goethe University. The goal of all projects is to improve the knowledge of the distribution of species. However, there are different approaches, e.g. targeted search for selected species, linking to historical data and integration into university teaching.
\end{abstract}

\section{Zusammenfassung}

Die kostenlose App „iNaturalist“ bringt interessierte Laien und Experten zusammen. Die App schafft einen niedrigschwelligen Zugang zur Biodiversitätserfassung und Pflanzenbestimmung, Experten sorgen ehrenamtlich für Qualitätssicherung. Auf verschiedenen räumlichen Ebenen wurden Projekte initiiert, z.B. durch die BVNH e.V., das Forschungsinstitut Senckenberg und die Goethe-Universität. Ziel aller Projekte ist die Verbesserung der Kenntnis der Verbreitung von Arten. Dabei gibt es jedoch unterschiedliche Ansätze, z.B. gezielt im Gelände nach ausgewählten Arten zu suchen, die Verknüpfung mit historischen Daten und die Einbindung in die universitäre Lehre.

\section{1. „Bürgerwissenschaftler“ sind gefragt}

Floristische Kartierungen, also die Erfassung der Pflanzenarten und ihrer Verbreitung in einem Gebiet, sind eine wichtige Grundlage für viele Ana-

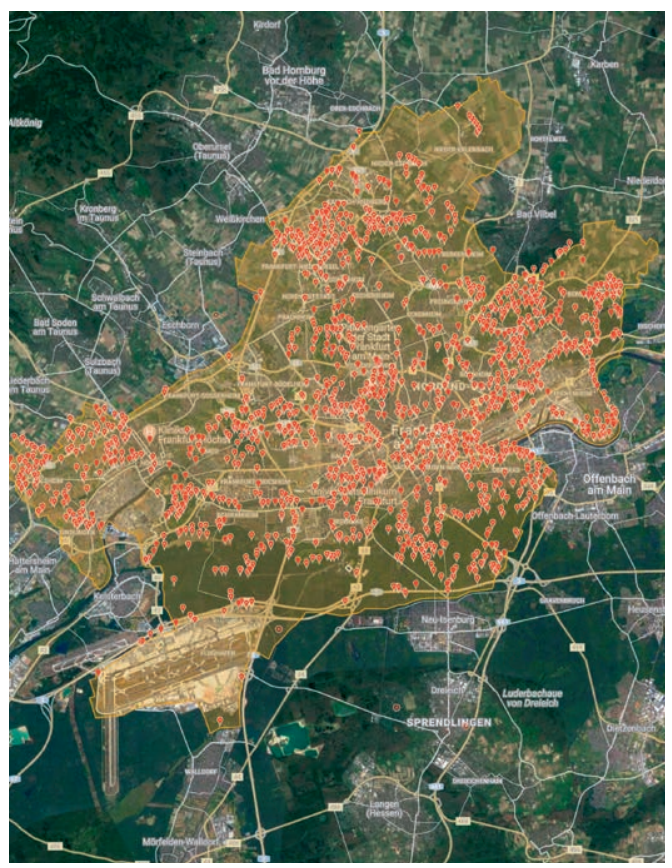

lysen und Bewertungen, etwa in Hinblick auf die Auswirkungen menschlicher Eingriffe, des Klimawandels oder die Erstellung von Roten Listen mit der Einschätzung der Gefährdung der Arten.

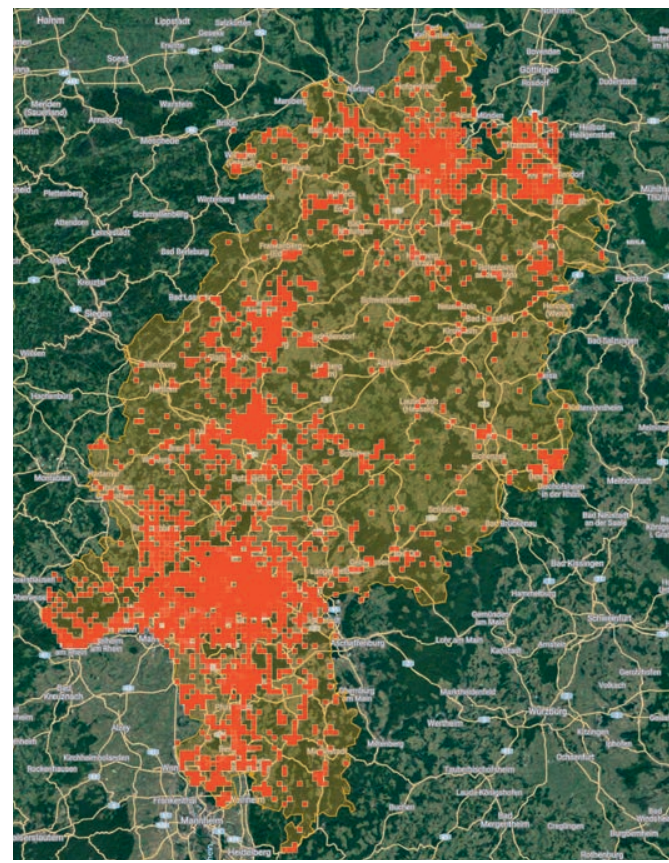

Abb. 1: Verteilung der Beobachtungen in Frankfurt (https://www.inaturalist.org/projects/flora-von-frankfurt, Stand 17.12.2020) und Hessen (https://www.inaturalist.org/projects/flora-und-rote-liste-von-hessen, Stand 17.12.2020). Es wird deutlich, dass die Beobachtungen sehr ungleich verteilt sind und bislang vor allem im städtischen Raum stattfinden. 
Dennoch ist es sehr schwierig, in der deutschen Forschungslandschaft Fördergelder für derartige Erfassungsprojekte zu erhalten. Seit Jahrzehnten stellt daher das Ehrenamt, also „Bürgerwissenschaftler" oder "Citizen scientists“, eine tragende Säule der meisten floristischen Kartierungen dar. Allerdings leidet dieser wichtige Pfeiler der Dokumentation und Erforschung der heimischen Pflanzenwelt an Überalterung und Nachwuchsmangel, und die Zahl derer, die in ihrer Freizeit Wildpflanzen erfassen wollen und dies auch können, sinkt ständig.

Wir versuchen daher, in verschiedenen Projekten die noch verbliebenen Kenner der heimischen Flora und naturinteressierte Laien zusammenzubringen. Dazu bieten Internet und Smartphones hervorragende Möglichkeiten. Wir nutzen dazu die Webseite und die App „iNaturalist“. Von zentraler Bedeutung sind dabei neuere Errungenschaften aus den Bereichen Weltraumtechnik und Künstliche Intelligenz (KI), die uns beide auch regelmäßig in den Tagesmedien begegnen: Die automatische Bestimmung und Dokumentation des eigenen Standortes durch Satellitenortung (GPS = Global Positioning System) und die Bestimmung von Pflanzen anhand von Fotos durch lernfähige Bilderkennungs-Software. Das eine musste früher an Karten abgelesen werden und konnte dennoch dann nur ungenau festgestellt werden, das andere, die Pflanzenkenntnis, war in langjähriger Beschäftigung mit der Flora erst mühsam zu erwerben.

\section{Kenntnislücken schließen}

Das Projekt „Flora und Rote Liste von Hessen“ wird von der Botanischen Vereinigung für Naturschutz in Hessen e. V. (BVNH) betreut. Mitglieder des Vereins erarbeiten die Roten Listen der Farn- und Samenpflanzen Hessens. Die 5. Auflage ist 2019 erschienen (Starke-Otтich et al. 2019). Bei der Erstellung wurden verschiedene Wissenslücken deutlich. Rote Listen sollten möglichst aktuell sein, das Bundesamt für Naturschutz empfiehlt die Aktualisierung etwa im Abstand von 10Jahren. Daher hat die BVNH das Projekt „Rote Liste 2030“ ins Leben gerufen. Die Zeit bis zur Herausgabe der 6. Auflage soll nun möglichst opti-

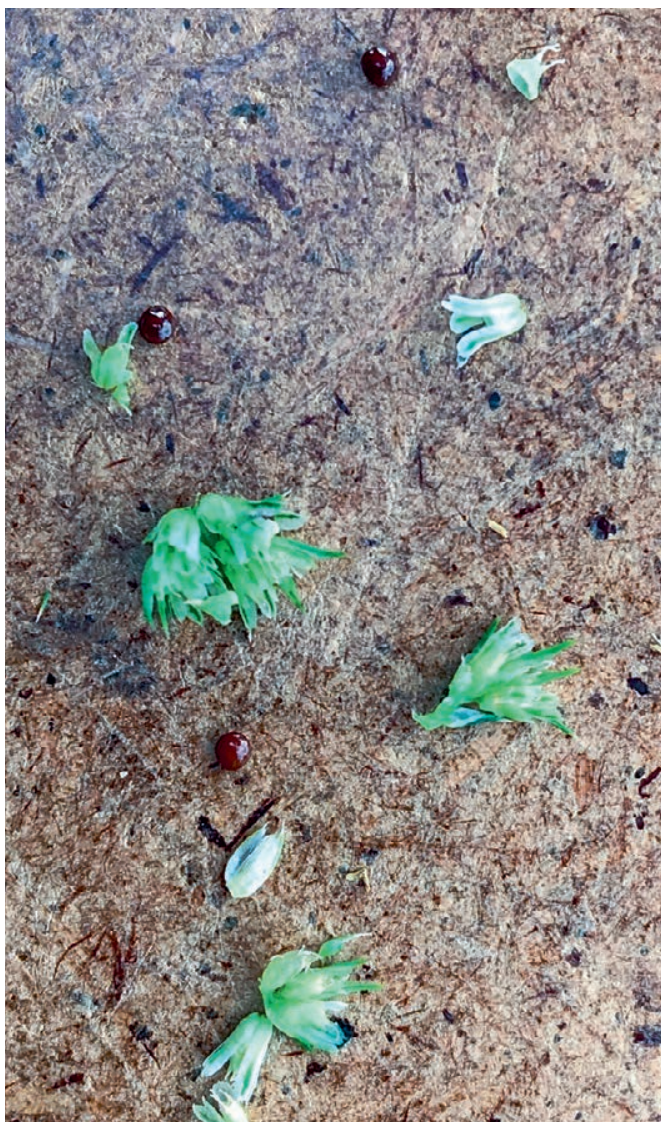

Abb. 2: Manchmal ist ein Schnappschuss zu wenig. Bei verschiedenen Pflanzengruppen werden für die sichere Bestimmung Details benötigt, wie hier beim Zurückgebogenen Fuchsschwanz (Amaranthus retroflexus).

(Foto: I. Starke-OtTich)

mal dafür genutzt werden, die bekannten Wissenslücken zu schließen. Ein Teil davon sind Projekte des Vereins, z. B. Auswertungen in Herbarien, gezielte Nachsuchen und Klärung taxonomischer (die Artabgrenzung betreffende) Fragestellungen.

Das iNaturalist-Projekt stellt die zweite wichtige Säule dar. Hier treffen interessierte Laien und Kenner der hessischen Pflanzenwelt aufeinander. Pflanzen mit dem Smartphone oder einer Digitalkamera fotografieren und hochladen kann jeder, Vorkenntnisse in der Pflanzenbestimmung sind dafür nicht nötig (aber natürlich hilfreich). Die künstliche Intelligenz, auf der die Pflanzenbestimmung bei iNaturalist basiert, ist bei dieser Artengruppe in Mitteleuropa bereits sehr gut trai- 


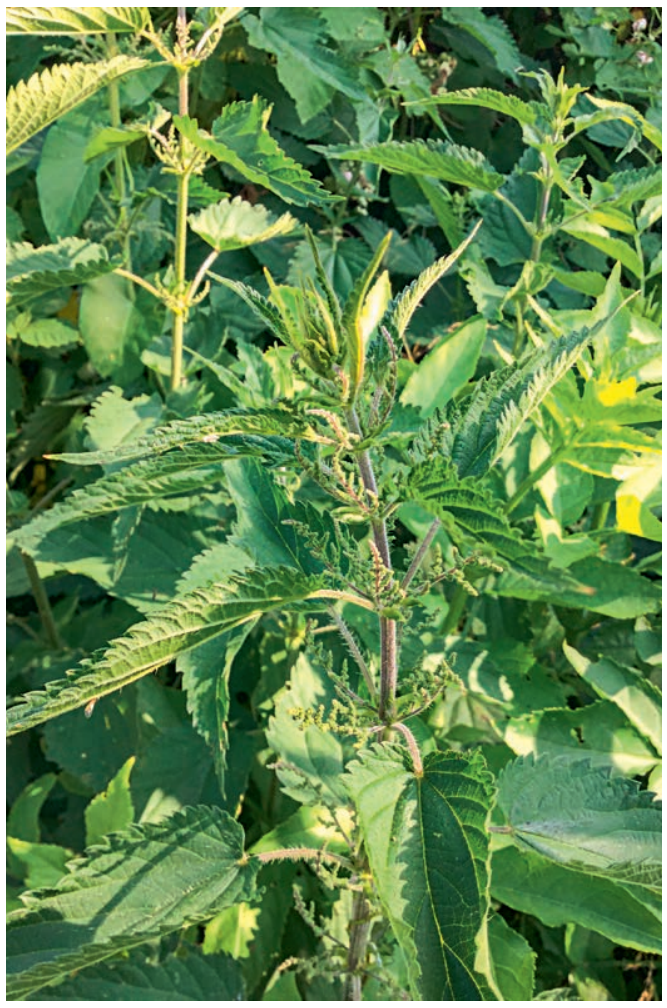

Abb. 3: Selten herbarisiert, oft fotografiert: Große Brennnessel (Urtica dioica). (Foto: I. StARKe-OTtich)

niert und trägt in den meisten Fällen zumindest zu einer hilfreichen Eingrenzung bei der Bestimmung bei (Schmidt \& Steinecke 2019), etwa auf Familien- oder Gattungsniveau. In der Regel gelingt die Bestimmung bis zur Art. Ausnahmen stellen noch für diesen Ansatz besonders problematischen Gruppen dar, z. B. Gräser, Seggen, Brombeeren oder Habichtskräuter. Bei diesen sind die kennzeichnenden Merkmale sehr klein, verborgen oder sehr ähnlich zwischen den Arten - das überfordert (noch) die einfache fotografische Dokumentation. Damit eine Beobachtung und Bestimmung „Forschungsqualität" erhält, muss sie durch mindestens eine weitere Person online bestätigt werden. Hier kommen die Pflanzenkenner ins Spiel, die ehrenamtlich die fotografierten Pflanzen ansehen und nachbestimmen. Ohne die Kenner, die wesentlich zur Qualitätssicherung beitragen, geht es auch hier nicht! Entscheidender Vorteil ist aber dabei, dass Pflanzenbilder und erste Bestimmung sofort einer großen, internationalen Community zugänglich sind. Dadurch kann es sein, dass ein Pflanzenfund von Kennern aus anderen Regionen oder Ländern in kürzester Zeit bestätigt (oder abgelehnt) wird. So gelingt es, einen täglich wachsenden Datenpool zur hessischen Flora zu generieren und unterschiedliche Personenkreise einzubinden. Interessierte können durch das Feedback von Experten nach und nach ihre Artenkenntnis und die fotografische Dokumentation durch Beachtung bestimmungsrelevanter Details verbessern. Und Kenner haben die Möglichkeit, bequem von zu Hause aus die Beobachtungen anzusehen und zu bearbeiten.

Der Projektansatz geht aber über diese ungerichtete Datensammlung hinaus. Das beschriebene Verfahren bietet die Möglichkeit, die Verteilung der dokumentierten Funde weniger „schief“" zu gestalten als bisherige Verfahren. Denn häufig werden Pflanzenfunde selektiv dokumentiert: Seltene Arten finden Interesse und werden notiert, häufige Sippen dagegen vielfach ignoriert. In Herbarien sind außerdem stechende, brennende oder unhandliche Pflanzen oft unterrepräsentiert. Dagegen ist bei iNaturalist die Große Brennnessel (Urtica dioica) mit 271 Beobachtungen die am häufigsten aus Hessen dokumentierte Art. Das Ziel des BVNH-Projektes ist es, mit Hilfe der über das Projekt vernetzten Naturbeobachter, die Datengrundlage zu den sogenannten mittelhäufigen Arten gezielt zu verbessern. In der Naturschutzarbeit der letzten Jahrzehnte standen insbesondere die sehr seltenen Arten im Fokus. Der Wissensstand zu deren Vorkommen ist in der Regel sehr gut. Daneben gibt es aber zahlreiche Arten, die zwar noch relativ weit in Hessen verbreitet sind, deren Bestände jedoch zurückgehen, ohne dass dies bislang hinreichend dokumentiert worden wäre, beispielsweise Arten des Grünlands. Zu einigen dieser mittelhäufigen Arten ist die aktuelle Verbreitung in Hessen schlichtweg nicht genau bekannt. Daher werden nun solche Arten als „Arten des Monats“ in dem Projekt in den Vordergrund gerückt, damit sie besondere Aufmerksamkeit erhalten und bevorzugt von den Beobachtern fotografiert und gemeldet werden. Als erste Art wurde im September 2020 das Gewöhnliche Seifenkraut (Saponaria officinalis) ausgewählt. Diese Daten 
sollen helfen, die Einschätzung zu eventuell vorhandenen Gefährdungen der Arten bei der Erstellung der nächsten Roten Liste zu verbessern. Grundsätzlich lassen sich mit der beschriebenen Methode viel schneller Daten sammeln als bisher. Eine so wesentlich verbesserte Datengrundlage verspricht ganz allgemein verbesserte Möglichkeiten zur Beurteilung von Veränderungen in der Verbreitung der (für die Erfassung mit dieser Methode geeigneten) Arten.

Bis Ende September 2020 waren bereits über 37000 Beobachtungen von wildwachsenden Pflanzen aus Hessen dokumentiert worden, davon sind allein im Monat September rund 4000 Beobachtungen eingegangen. Ungefähr ein Viertel der Beobachtungen wartet noch auf die abschließende Bestimmung. Dies ist eine gute Gelegenheit, um vorhandene Kenntnisse in das Projekt einzubringen, auch wenn vielleicht Zeit oder Möglichkeiten für eigene Exkursionen fehlen. Außerdem ist es eine schöne Beschäftigung für die Wintermonate, wenn es weniger Pflanzen im Gelände zu beobachten gibt. Nimmt man sich gezielt einzelne Arten vor und entsprechende Bestimmungsliteratur zur Hand, kann man auch seine eigenen Kenntnisse auf diese Weise trainieren, während man gleichzeitig bei der Qualitätssicherung der Datensammlung hilft. Es gibt also viele Möglichkeiten, sich in das Projekt einzubringen!

\section{Stadtflora online}

Das Projekt „Flora von Frankfurt“ wird vom Forschungsinstitut Senckenberg betreut. Senckenberg ist bereits 2009 mit der Webseite www.flora-frankfurt.de online gegangen und bietet dort eine Fülle von Frankfurt-spezifischen, auch historischen Informationen zur Pflanzenwelt (BöNSEL et al. 2010). Zudem gab es die Möglichkeit, Funde zu melden. Softwareprobleme und ein Hacker-Angriff legten vor einiger Zeit diese Funktion der Seite lahm. Derzeit wird daran gearbeitet, die Software zu aktualisieren. In der Zwischenzeit können aber neue Funde über iNaturalist dokumentiert werden. Es ist geplant, diese Daten in Zukunft mit dem bei Senckenberg vorhandenen Datensatz zur Flora von Frankfurt, der immerhin über 125000

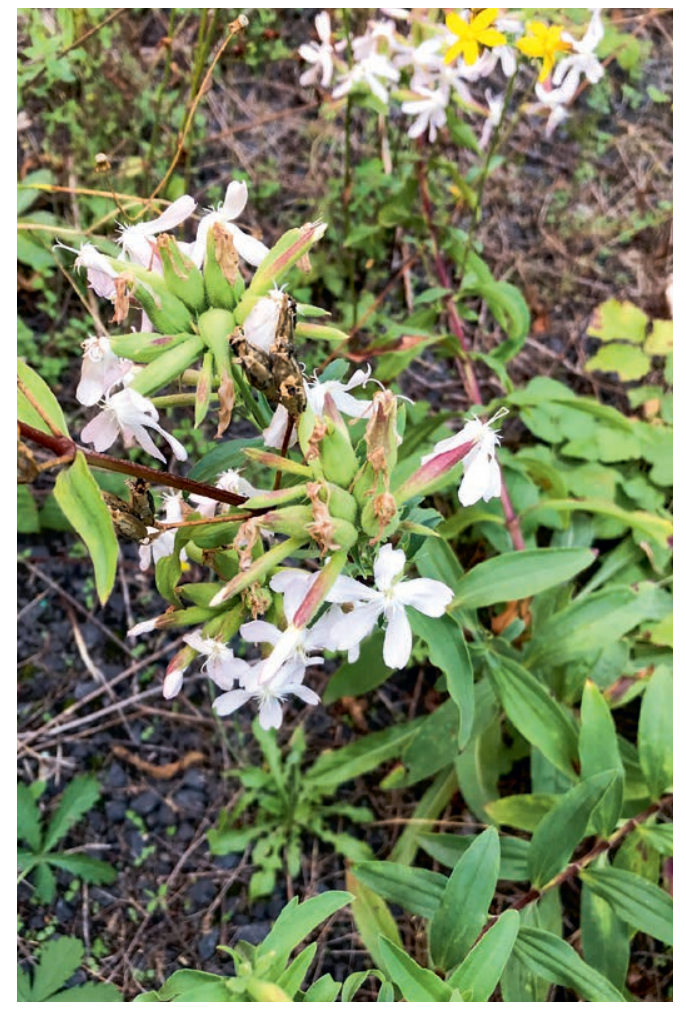

Abb. 4: Um die Kenntnis der Verbreitung in Hessen zu verbessern wurde das Gewöhnliche Seifenkraut (Saponaria officinalis) als erste „Art des Monats“ ausgewählt.

(Foto: I. STARKe-OTtich)

Einträge umfasst und auch zahlreiche historische Daten enthält, zu verknüpfen, um die Kenntnis der Frankfurter Pflanzenwelt weiter zu verbessern.

Für das Projekt „Flora von Frankfurt" werden grundsätzlich Informationen zu allen in Frankfurt wild vorkommenden Pflanzenarten gesammelt. Entsprechend der Arbeitsschwerpunkte der Mitglieder der Abteilung Botanik und Molekulare Evolutionsforschung bei Senckenberg. gilt ein besonderes Augenmerk der Erfassung von Neophyten (also pflanzlichen Neueinwanderern) sowie seltenen bzw. gefährdeten Arten im Stadtgebiet. Aber natürlich sind Daten über alle Arten aus Frankfurt willkommen! Derzeit führt der durch seine blau-violetten Blüten sehr attraktive Gemeine Natternkopf (Echium vulgare) mit 122 Beobachtungen die Statistik in Frankfurt an. Wichtig ist bei den Beobachtungen, dass es sich um wild- 


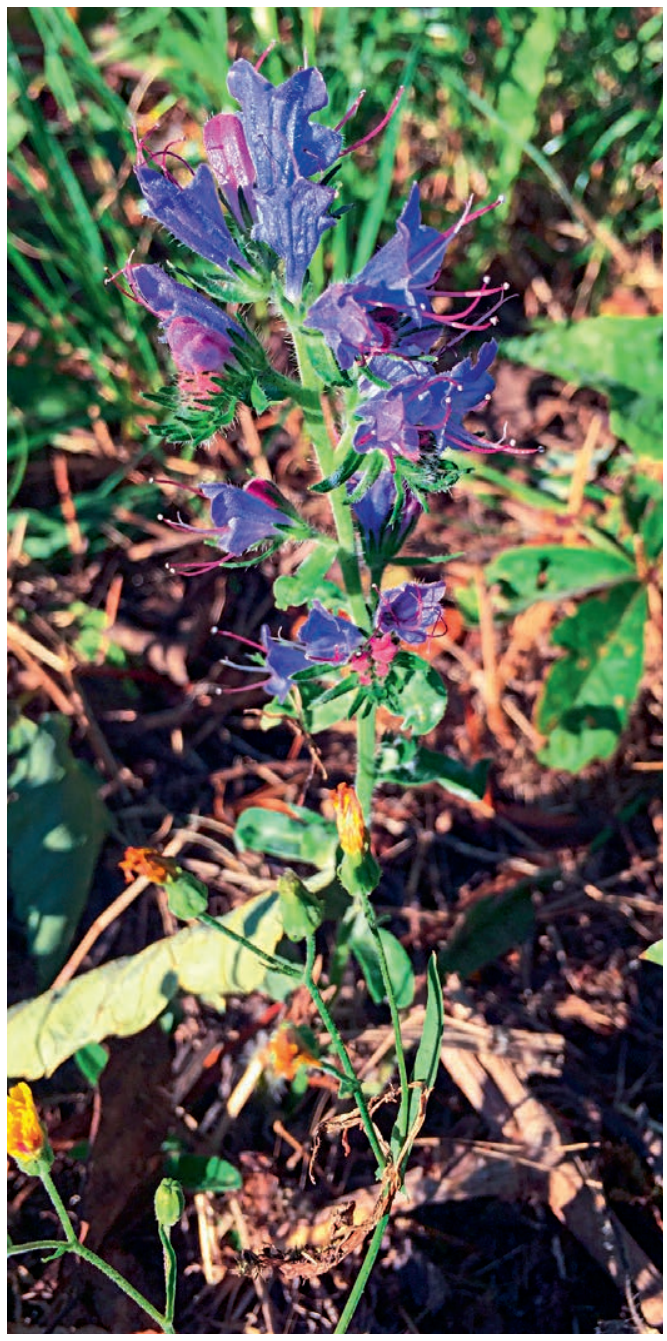

Abb. 5: Der Gewöhnliche Natternkopf (Echium vulgare) ist bei iNaturalist bislang die am häufigsten in Frankfurt fotografierte Pflanzenart. (Foto: I. Starke-Otтich)

wachsende Arten handelt, also nicht um gepflanzte Parkbäume, Garten- oder Balkonpflanzen, Ansaat-Mischungen für Insekten etc. Auch solche Exemplare kann man fotografieren und hochladen und die App als Bestimmungshilfe nutzen, muss dann aber daran denken, das Häkchen im Feld „In Haltung/kultiviert“ zu setzen, damit diese Beobachtungen nicht bei Auswertungen als Wildpflanze eingehen.

Die Gebiete der beiden Projekte überschneiden sich, denn da Frankfurt in Hessen liegt, gehen alle
Beobachtungen zur „Flora von Frankfurt“ automatisch auch in das Projekt „Flora und Rote Liste von Hessen “ ein. Derzeit machen die Frankfurter Beobachtungen fast ein Drittel aller Nachweise aus Hessen aus. Auch aus anderen Städten wie Gießen, Marburg und Kassel, sowie deren Umgebung werden relativ viele Beobachtungen beigetragen, vergleichsweise wenige aus dünner besiedelten Regionen wie Vogelsberg und Rhön. Aber auch innerhalb Frankfurts sind die Beobachtungen nicht gleichmäßig verteilt. Denn es gibt weitere Projekte, die sich nur auf Teile von Frankfurt beziehen. So gehen knapp ein Viertel der Frankfurter Nachweise auf das Konto von Projekten am Campus Riedberg.

\section{Smartphone im Studium}

Mit der Möglichkeit, bei iNaturalist bestimmte räumliche Bereiche als „Projekte“ zu definieren, für die dann auch Artenlisten abgefragt und ausgelesen werden können, kann zusätzliche Motivation für die Beschäftigung mit der Biodiversität erzeugt werden. Diese Möglichkeit kam angesichts der Corona-Pandemie gerade recht, die dafür sorgte, dass 2020 für viele Angebote des Biologie-Studiums neue Konzepte gefunden werden mussten. So wurden an der Goethe-Universität von den Bereichen „Diversität der Organismen“ und „Didaktik der Biologie“ verschiedene Projekte am Campus Riedberg und im Wissenschaftsgarten auf den Weg gebracht. Smartphone und App werden dadurch bei der Erarbeitung der Grundkenntnisse der Artenkenntnis heimischer Pflanzen, Tiere und Pilze zu sehr nützlichen Hilfsmitteln für die Studierenden. Gleichzeitig erzeugen sie Daten, die wiederum in andere Projekte einfließen können. Aktuell ist - zumindest bei iNaturalist - der Campus Riedberg wohl das am besten dokumentierte Gebiet Hessens.

\section{Fazit}

Lange galten Smartphone und Internet als Motoren der zunehmenden Naturentfremdung. Die Welt der traditionellen Pflanzenkenner schien unvereinbar mit der Realität moderner Studenten. Inzwischen ist die Technik jedoch so weit gereift, dass man sie nutzen kann, um Brücken zu schlagen und Menschen unterschiedlicher Alters- und Interes- 


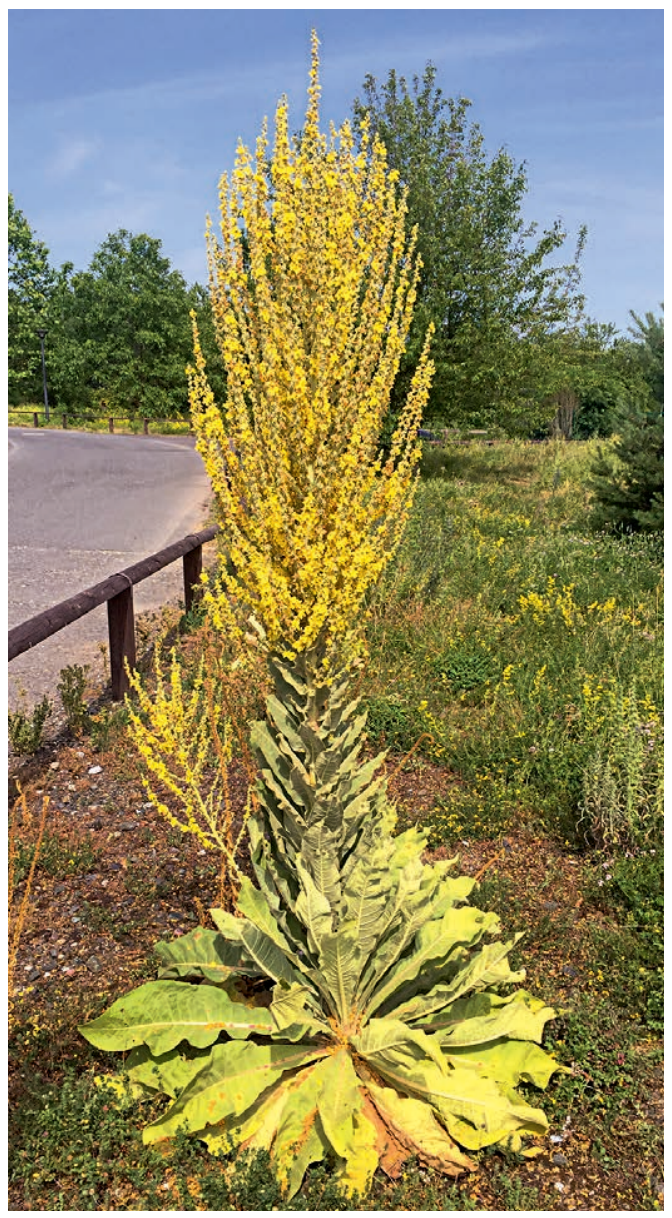

Abb. 6: Eine stattliche Pracht-Königskerze (Verbascum speciosum) wurde am Campus Riedberg dokumentiert. (Foto: G. ZiZKa)

sensgruppen miteinander zu vernetzen. Bereits seit mehreren Jahren werden auf verschiedenen Portalen Beobachtungsdaten gesammelt und liefern inzwischen wichtige Daten für die Wissenschaft, z.B. im Bereich der Ornithologie. Viele davon haben den Fokus auf nur einer Organismengruppe, manche sind von Experten für Experten gemacht. Wir haben uns für die genannten Projekte aus der Vielfalt der Angebote für iNaturalist entschieden, weil die Dokumentation jedes einzelnen Fundes mit mindestens einem Foto die Daten überprüfbar macht - aus wissenschaftlicher Sicht ein wichtiges Kriterium! Der Einstieg ist niedrigschwellig und die App bietet für jeden was: Man kann sie gleichermaßen für wissenschaftliche Projekte als auch dafür benutzen, eine Kübelpflanze oder die Spinne an der Zimmerdecke zu bestimmen. Daneben gibt es auch einen Wettbewerbs-Charakter, der an Computerspiele erinnert. Rankings listen ähnlich einem Highscore beim Spiel auf, wer die meisten Beobachtungen in einem Projekt gemacht hat und wer die meisten Arten gefunden hat. Manche Nutzer setzen sich Ziele, wie viele Arten sie in einem Jahr finden wollen. Viele verschiedene Arten zu finden, das gelingt nur, wenn man sich mit der Natur auch auseinandersetzt, Exkursionen in verschiedene Lebensräume unternimmt und ganz genau hinschaut - heute eben mit dem Smartphone in der Hand.

\section{Literatur}

Bönsel, D., Brunken, U., Gregor, T., Malten, A., OtTICH, I. \& ZIZKA, G. 2010: Frankfurter Flora geht ins Netz. Natur und Museum 140 : 50-57.

Schmidt, M. \& Steinecke, H. 2019: Heimische Pflanzen mit dem Smartphone bestimmen - ein Praxistest. - Palmengarten 83: 138-140.

Starke-Ottich, I., Gregor, T., Uebeler, M., Frede, A., Kubosch, R., Mahn, D., Barth, U., Gregor, T., Bönsel, D., Böger, K., Hodvina, S., Cezanne, R., Hemm, K., Gottschlich, G., Jansen, W. \& Blatt, H. 2019: Rote Liste der Farn- und Samenpflanzen Hessens. 5. Fassung. Hessisches Landesamt für Naturschutz, Umwelt und Geologie.

\section{Internetseiten}

http://www.bvnh.de/

https://www.inaturalist.org/projects/flora-und-rote-liste-vonhessen

http://www.flora-frankfurt.de

https://www.inaturalist.org/projects/flora-von-frankfurt

https://www.inaturalist.org/projects/pflanzen-tiere-und-pilzeam-campus-riedberg-der-goethe-universitat-frankfurt

\section{Anschriften der Autorin und der Autoren}

Dr. Indra Starke-Ottich \& Prof. Dr. Georg Zizka, Senckenberg Forschungsinstitut und Naturmuseum Frankfurt, Senckenberganlage 25, 60325 Frankfurt, E-Mail: indra.starke-ottich@senckenberg.de bzw. georg.zizka@senckenberg.de

Dr. Marco Schmidt, Palmengarten Frankfurt, Siesmayerstraße 61, 60323 Frankfurt,

E-Mail: marco.schmidt@stadt-frankfurt.de 